\title{
Effect of Wilms tumor histology on response to neoadjuvant chemotherapy
}

\section{Taskinen, Seppo}

2019-04

Taskinen , S , Leskinen , O , Lohi , J , Koskenvuo , M \& Taskinen , M 2019 , ' Effect of Wilms tumor histology on response to neoadjuvant chemotherapy ' , Journal of Pediatric Surgery , vol. 54 , no. 4 , pp. 771-774 . https://doi.org/10.1016/j.jpedsurg.2018.05.010

http://hdl.handle.net/10138/301857

https://doi.org/10.1016/j.jpedsurg.2018.05.010

cc_by_nc_nd

acceptedVersion

Downloaded from Helda, University of Helsinki institutional repository.

This is an electronic reprint of the original article.

This reprint may differ from the original in pagination and typographic detail.

Please cite the original version. 


\section{Accepted Manuscript}

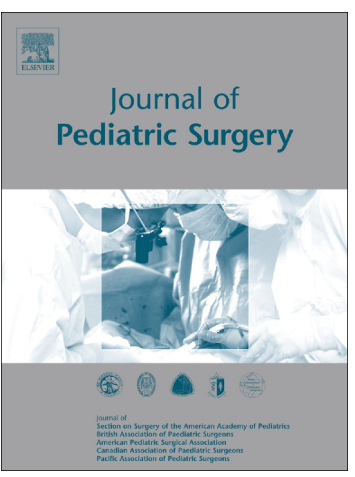

Seppo Taskinen, Outi Leskinen, Jouko Lohi, Minna Koskenvuo, Mervi Taskinen

PII:

S0022-3468(18)30336-1

DOI:

doi:10.1016/j.jpedsurg.2018.05.010

Reference:

YJPSU 58695

To appear in:

Received date: $\quad 23$ February 2018

Revised date: $\quad 8$ April 2018

Accepted date: $\quad 15$ May 2018

Please cite this article as: Seppo Taskinen, Outi Leskinen, Jouko Lohi, Minna Koskenvuo, Mervi Taskinen, Effect of Wilms tumor histology on response to neoadjuvant chemotherapy. The address for the corresponding author was captured as affiliation for all authors. Please check if appropriate. Yjpsu(2018), doi:10.1016/j.jpedsurg.2018.05.010

This is a PDF file of an unedited manuscript that has been accepted for publication. As a service to our customers we are providing this early version of the manuscript. The manuscript will undergo copyediting, typesetting, and review of the resulting proof before it is published in its final form. Please note that during the production process errors may be discovered which could affect the content, and all legal disclaimers that apply to the journal pertain. 
Effect of Wilms tumor histology on response to neoadjuvant chemotherapy

Seppo Taskinen ${ }^{1}$, Outi Leskinen ${ }^{2}$, Jouko Lohi ${ }^{3}$, Minna Koskenvuo ${ }^{4}$, Mervi Taskinen ${ }^{4}$

${ }^{1}$ Department of Pediatric Surgery, Children's Hospital, Helsinki University Hospital, Helsinki, Finland

${ }^{2}$ Department of Radiology, Helsinki University Hospital, Helsinki, Finland

${ }^{3}$ Department of Pathology, Helsinki University Hospital, Helsinki, Finland

${ }^{4}$ Division of Pediatric Hematology/Oncology and Stem Cell Transplantation, Children's Hospital, Helsinki University Hospital, Helsinki, Finland

Key words: Wilms' tumor; Chemotherapy; Histology; Tumor volume

An abstract has been presented in the annual congress of European Society for Pediatric Urology 2017

Correspondence

Seppo Taskinen

Children's Hospital, Helsinki University Hospital

Stenbäckinkatu 11

00290 Helsinki, Finland

tel: +358504272542

fax: + 358947175314

e-mail: seppo.taskinen@hus.fi; seppo.taskinen@gmail.com 
Purpose. To evaluate the association between Wilms tumor histology at diagnosis and the change in Wilms' tumor volume during preoperative chemotherapy.

Methods. We included all the 52 patients operated for Wilms tumor at 1988-2015, who had both pathology samples and either CT or MRI-images before and after preoperative chemotherapy, available for re-evaluation.

Results: The median tumor volume was 586ml (IQR 323-903) at diagnosis. The median change in tumor volume was $-68 \%$ (IQR - 85-40, $\mathrm{p}<0.001$ ) and the proportion of tumor necrosis $85 \%$ (IQR 24-97), after preoperative chemotherapy. There was a correlation between blastemal cell content in pre-chemotherapy needle biopsy sample and the reduction in tumor volume $(\mathrm{Rho}=-0.452, \mathrm{p}=0.002)$. High stromal and epithelial cell contents in CNB samples were associated with the lesser change in tumor volume $(\mathrm{Rho}=0.279, \mathrm{p}=0.006$ and $\mathrm{Rho}=0.300, \mathrm{p}=0.038$ respectively). Reduction of tumor volume and the proportion of tumor necrosis after chemotherapy were associated $(\mathrm{Rho}=-0.502, \mathrm{p}<0.001)$. The actual viable tumor volume decreased in median by $97 \%$ (IQR 65-100), and the decrease could be seen in all cellular components. In three patients, the tumor volume increased more than $10 \%$ during the preoperative chemotherapy. Two of them had anaplastic tumor in the nephrectomy specimen. Conclusion: Wilms tumor total and viable tumor volumes reduced by $68 \%$ and $97 \%$ with preoperative chemotherapy, respectively. High proportion of blastemal cells in CNB was associated with greatest decrease in Wilms tumor volume. Increase in tumor volume during preoperative chemotherapy may indicate anaplastic tumor and prolonging of preoperative therapy should be avoided. 
Type of study: Retrospective review

Level of evidence: Level III

Keywords

Wilms' tumor; chemotherapy; tumor volume; histology 


\section{Effect of Wilms tumor histology on response to neoadjuvant chemotherapy}

Wilms tumor is often large and fragile. Consequently, large tumors are prone to rupture during surgery $[1,2]$. Preoperative chemotherapy is systematically used in The International Society of Pediatric Oncology (SIOP) and United Kingdom Children's Cancer Study Group (UKCCSG) treatment protocols and, in the case of inoperable tumors, by others too[3-5]. Following preoperative chemotherapy, the tumor size often shrinks enabling a safer operation with a decreased risk for tumor rupture [6]. In addition, the down-staging of the tumor with preoperative chemotherapy has been suggested to spare $20 \%$ of the patients from radiotherapy or doxorubicin by UKW-3 study [7]. In a previous SIOP-9 study, tumors with stromal or epithelial predominance after chemotherapy had reduced less in volume than the tumors with necrotic, regressive or blastemal predominance [8]. However, association between pre-chemotherapy histology and tumor volume change during chemotherapy has not been investigated in detail previously.

To confirm diagnosis and to direct the most appropriate neoadjuvant regimen according to histology, cutting needle biopsy $(\mathrm{CNB})$ has been used at diagnosis in our center as well as in some other centers [9-12]. This allows us to compare the histological findings of the primary biopsy to the tumor volume changes during the preoperative chemotherapy, which is the primary goal of our study. In addition, we evaluated the association between postchemotherapy findings and the effect of the length of preoperative chemotherapy to the tumor volume change. Our hypothesis is that prechemotherapy histology has impact on the tumor response. 


\section{Methods}

\subsection{Data source and subjects}

The study cohort was identified by a retrospective review of the surgical database for patients operated for renal tumors in 1988 through 2015 in the Children's Hospital, University of Helsinki. The institutional ethics committee had approved the study. Altogether 90 patients were operated because of renal tumor and 70 of them had had diagnostic CNB.

Diagnostic CNB has been part of our clinical work up of renal tumors since the initial report . [9] CNB was not performed in case of small, by imaging intrarenal tumors, cystic tumors and tumors with active bleeding. . CNB was taken by using ultrasound guidance from the back or flank to avoid peritoneum, and at least two acceptablequality cylinders were collected. Originally, manual core needles were used but during the last 20 years automatic cutting needles (at present BioPince® 18ga, Argon Medical Devices, Tx, USA) have been used.

We excluded six patients whose pathology slides could not be found for re-evaluation and five patients with a non-Wilms tumor. An additional seven patients were excluded due to lacking either pre- or postchemotherapy CT- or MRI-images. Accordingly, we included to the final analysis all the 52 Wilms tumor patients who had both original preoperative CNB and the histological samples from nephrectomy available as well as CT- or MRI-images from both before and after chemotherapy. The pathology slides were reevaluated by an experienced pathologist (JL) to confirm the diagnosis and to classify the response to preoperative chemotherapy according to current guidelines [13]. The structured histological evaluation included the estimation of the degree of necrosis (necrotic $=100 \%$ 
necrosis, regressive $=66-99 \%$ necrosis, poor response $=$ necrosis $<66 \%$ ), proportions of the cellular components of Wilms tumor (epithelial, blastemal, stromal) were expressed as a percent of the total surface area of the viable tissue of the tumor, and the presence of anaplasia (focal or diffuse) [13]. Operative staging of the tumors was done by SIOP WT 2001 staging criteria [13]. Images were re-evaluated by a radiologist (OL) for the exact tumor volumes with ellipsoid formula $(0.523 \mathrm{x}$ a $\mathrm{x}$ b x c) [14]. Viable tumor volume in nephrectomy specimen was calculated by reducing the proportion of necrotic tumor (determined from histologic sample) from actual tumor volume in imaging study. At diagnosis the whole tumor was considered to be viable.

\subsection{Chemotherapy regimen}

The chemotherapy regimen used preoperatively was based on dactinomycin and vincristine for 2 weeks -3 months intensified with alkylators or anthracycline in patients with metastases or biopsy showing either anaplasia or in the early series if the histology was composed mostly of blastemal cells.[15] The length of chemotherapy was often increased from the standard one month if the reduction of tumor volume was considered insufficient, especially in the early series.

\subsection{Statistical analysis}

The association between different cellular components on CNB and the degree of necrosis or reduction in tumor volume was evaluated with Spearman correlation. The change in tumor volume was evaluated with Wilcoxon signed rank test. Continuous variables are expressed as medians and interquartile ranges (IQR) or ranges. For the analyses Statview ${ }^{\circledR}$ 5.0.1, SAS Institute Inc. was used. P-value $<0.05$ was considered significant. 


\section{Results}

\subsection{Patient characteristics}

Twenty-three of the 52 patients were male. The median age at diagnosis was 3.4 years (IQR 2.0-5.0). The tumor was located on the right or left side, or bilateral in $28(54 \%), 21(40 \%)$ and three $(6 \%)$ patients, respectively. In the cases of bilateral tumor, only the larger tumor was evaluated.

\subsection{Histology of the specimens}

The proportion of blastemal, stromal and epithelial components in the diagnostic CNB were 60\% (IQR 25-86), 20\% (IQR 0-50) and 2\% (IQR 0-10), respectively. The proportion of necrosis was $85 \%$ (IQR 24-97) in the nephrectomy samples. The nephrectomy specimens were classified as fully necrotic and regressive in $5(10 \%)$ and 23 (44\%) cases, respectively. The tumors with necrosis less than $66 \%$ were mixed in $8(15 \%)$, blastemal type in 14 $(8 \%)$, stromal type in $8(15 \%)$ and epithelial type in $2(4 \%)$. Two cases $(4 \%)$ were nephrobastomas with diffuse anaplasia. Operative local stage 1 was confirmed in 30, stagem 2 in nine and stage 3 in 13 cases.

\subsection{Change in tumor volume}

The median tumor volume at diagnosis was $583 \mathrm{ml}$ (IQR 368-903) and the greatest dimension was $127 \mathrm{~mm}$ (IQR 99-144). The median volume change during the preoperative chemotherapy was $-68 \%$ (IQR-85- -40 , range $-98-+265, \mathrm{p}<0.001$ ). The more the tumor volume reduced during the preoperative chemotherapy, the larger the tumor necrosis was in the nephrectomy specimen (Rho=-0.502, p<0.001, Fig. 1). In two cases, tumor volume did not decrease despite of complete or near complete tumor necrosis. In three patients, the tumor volume increased more than $10 \%$ during the preoperative chemotherapy (Fig. 2). Two 
of them had anaplastic tumor (increase in tumor volume 26 and 265\%) and the third showed stromal predominance in the nephrectomy specimen (volume increase $34 \%$ ).

The proportion of blastemal component in CNB correlated with the reduction in tumor volume during the preoperative chemotherapy ( $\mathrm{p}=0.002$, Fig. 3). Accordingly, the higher the content of stromal or epithelial component in the CNB, the less was the reduction in tumor volume $(\mathrm{p}=0.053$ and $\mathrm{p}=0.038$ respectively $)$.

When the degree of tumor necrosis and the change in tumor volume were combined the actual decrease in viable tumor volume was in median 97\% (IQR 65-100) and the reduction could be seen in all cellular components (99\% (IQR 93-100) in blastemal, 98\% (IQR 55-100) in stromal and 85\% (IQR 27-99) in epithelial component). In nine patients treated with 2-3months' preoperative chemotherapy, CT or MRI images were available also after 4-6-weeks' chemotherapy. In six of them tumor shrank more than $10 \%$, the tumor size remained unchanged in one patient and in two patients (one with anaplastic tumor and the other with stromal type tumor) the tumor grew with prolonged chemotherapy.

\section{Discussion}

The median reduction in Wilms tumor volume was $68 \%$ with pre-operative chemotherapy. A high proportion of blastemal cells in diagnostic CNB was associated with a significant decrease in Wilms tumor volume, whereas high percentage of stromal or epithelial cells was associated with poor reduction in tumor volume during the pre-operative chemotherapy. However, the actual viable volume of all these three components decreased during the 
chemotherapy. Tumor growth was rare during the pre-operative chemotherapy and it was associated with diffuse anaplasia in two of the three cases.

In previous studies evaluating the effect of preoperative chemotherapy on tumor volume no comparisons were made to prechemotherapy histology in contrast to our study. In SIOP-9 study with 1052 patients, the median tumor volume reduction was $62 \%$ with preoperative chemotherapy [16]. An increase in tumor volume was observed in $12 \%$ of the patients. In SIOP-9 /GPOH study, analyzing the association between tumor histology in nephrectomy samples and the change in tumor volume during preoperative chemotherapy, more than $40 \%$ of decrease in volume was common in necrotic, regressive, mixed or blastemal type tumor in nephrectomy specimens but rare in epithelial and stromal type tumors [8]. Our results are in accordance with those results. In a cohort of patients treated in UKW-3 study, all five high risk tumors reduced in tumor volume (in median by 79\%). However, among patients belonging to intermediate risk group (epithelial, stromal, regressive or focal anaplasia type in nephrectomy specimen) only $10 / 15$ had reduction in tumor volume whereas $5 / 15$ had increase in tumor volume [17]. Similarly, in a study from Belgium, in which most tumors belonged to the intermediate group, the mean reduction of tumor volume was $61 \%$ in $12 / 17$ cases, whereas in the remaining five cases tumor volume increased in mean by $43 \%$ [18]. When applying the UK study risk classification criteria to our study, six high risk tumors with blastemal predominance after chemotherapy had comparable decrease in tumor volume as in the UK study. However, in contrast to the UK study, both tumors with diffuse anaplasia in our series were chemoresistant and grew despite the use of preoperative chemotherapy. Furthermore, in our study only one (stromal type) of 42 intermediate risk tumors continued to grow despite of chemotherapy. 
Our study has several limitations rising from the retrospective nature of this study. In addition, the calculation of viable tumor volume was based on assumption that before chemotherapy the whole tumor is viable, which is in many cases apparently not true. In post chemotherapy samples the degree of necrosis was based completely on histopathology evaluation. Accordingly, it is possible that in some cases the reported decrease in viable tumor is overestimated. However, all the histological samples were reevaluated by an experienced pathologist and all the imaging studies were evaluated by a radiologist to cover the possible inter-examiner bias of the histological findings and tumor volumes. In addition, because this study analyses the results from the last 30 years, there have been changes in both chemotherapy and the length of preoperative therapy.

\section{Conclusions}

Primary chemotherapy before nephrectomy reduces the median total volume 68 percent and the viable Wilms tumor volume 97 percent. The tumors that do not shrink during the preoperative chemotherapy more often have a predominant epithelial or stromal component remaining. Tumor growth during chemotherapy may indicate anaplastic tumor and prolonging chemotherapy should be avoided.

\section{Conflict of interest statement}

None 


\section{References}

[1] Barber TD, Derinkuyu BE, Wickiser J, et al. Wilms tumor: preoperative risk factors identified for intraoperative tumor spill. J Urol. 2011;185:1414-8.

[2] Ritchey ML, Shamberger RC, Haase G, et al. Surgical complications after primary nephrectomy for Wilms' tumor: report from the National Wilms' Tumor Study Group. J Am Coll Surg. 2001;192:63-8.

[3] Nakamura L, Ritchey M. Current management of Wilms' tumor. Curr Urol Rep. 2010;11:58-65.

[4] Irtan S, Ehrlich PF, Pritchard-Jones K. Wilms tumor: "State-of-the-art" update, 2016. Semin Pediatr Surg. 2016;25:250-6.

[5] Refaie HD, Sarhan M, Hafez A. Role of CT in assessment of unresectable Wilms' tumor response after preoperative chemotherapy in pediatrics. ScientificWorldJournal. 2008;8:6619.

[6] Powis M, Messahel B, Hobson R, et al. Surgical complications after immediate nephrectomy versus preoperative chemotherapy in non-metastatic Wilms' tumour: findings from the 1991-2001 United Kingdom Children's Cancer Study Group UKW3 Trial. J Pediatr Surg. 2013;48:2181-6.

[7] Mitchell C, Pritchard-Jones K, Shannon R, et al. United Kingdom Cancer Study Group. Immediate nephrectomy versus preoperative chemotherapy in the management of nonmetastatic Wilms' tumour: results of a randomised trial (UKW3) by the UK Children's Cancer Study Group. Eur J Cancer. 2006;42:2554-62.

[8] Weirich A, Leuschner I, Harms D, et al. Clinical impact of histologic subtypes in localized non-anaplastic nephroblastoma treated according to the trial and study SIOP-9/GPOH. Ann Oncol. 2001;12:311-9.

[9] Saarinen UM, Wikström S, Koskimies O, et al. Percutaneous needle biopsy preceding preoperative chemotherapy in the management of massive renal tumors in children. J Clin Oncol. 1991;9:406-15.

[10] Dykes EH, Marwaha RK, Dicks-Mireaux C, et al. Risks and benefits of percutaneous biopsy and primary chemotherapy in advanced Wilms' tumour. J Pediatr Surg. 1991;26:610-2. [11] McLorie GA, McKenna PH, Greenberg M, et al. Reduction in tumor burden allowing partial nephrectomy following preoperative chemotherapy in biopsy proved Wilms tumor. $\mathbf{J}$ Urol. 1991;146:509-13.

[12] Sköldenberg EG, Jakobson A, Elvin A, et al. Pretreatment, ultrasound-guided cutting needle biopsies in childhood renal tumors. Med Pediatr Oncol. 1999;32:283-8.

[13] Vujanić GM, Sandstedt B. The pathology of Wilms' tumour (nephroblastoma): the International Society of Paediatric Oncology approach. J Clin Pathol. 2010;63:102-9.

[14] Al-Shanafey S, Yanchar NL, Schmidt MH, et al. CT-estimated volume of Wilms tumor can predict weight. J Pediatr Hematol Oncol. 2005;27:311-3.

[15] Taskinen S, Lohi J, Koskenvuo M, Taskinen M. Evaluation of effect of preoperative chemotherapy on Wilms' tumor histopathology. J Pediatr Surg. 2017 Oct 6. pii: S00223468(17)30619-X. doi: 10.1016/j.jpedsurg.2017.10.002. [Epub ahead of print] PMID: 29074135

[16] Godzinski J, van Tinteren H, de Kraker J, et al. SIOP Nephroblastoma Trial \& Study Committee. Nephroblastoma: does the decrease in tumor volume under preoperative chemotherapy predict the lymph nodes status at surgery? Pediatr Blood Cancer.

2011;57:1266-9. 
[17] Olsen ØE, Jeanes AC, Sebire NJ, et al. Changes in computed tomography features following preoperative chemotherapy for nephroblastoma: relation to histopathological classification. Eur Radiol. 2004;14:990-4.

[18] Bogaert GA, Heremans B, Renard M, et al. Does preoperative chemotherapy ease the surgical procedure for Wilms tumor? J Urol 2008; 1869-75. 


\section{Figure legends}

Fig. 1. Degree of necrosis and the change in tumor volume after pre-operative chemotherapy in 50 patients with Wilms tumor. The patients with anaplastic tumors were excluded.

Fig.2. Wilms tumor histological classification in 52 nephrectomy specimens and the change in tumor volume during preoperative chemotherapy.

Fig. 3. The change in tumor volume during preoperative chemotherapy and the proportion of different cellular components in $\mathrm{CNB}$ at diagnosis. 


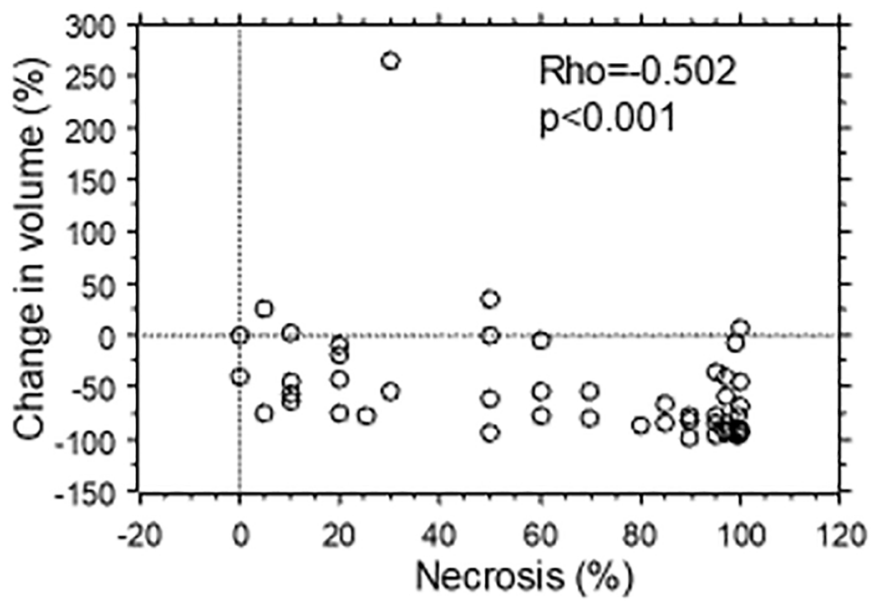

Figure 1 


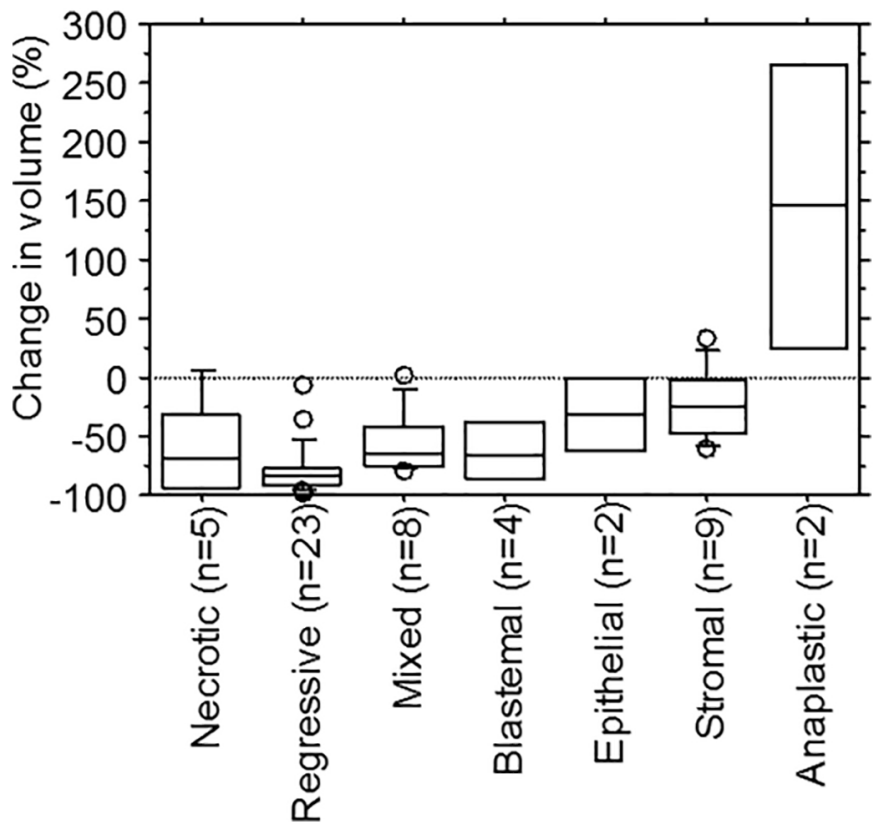

Figure 2 

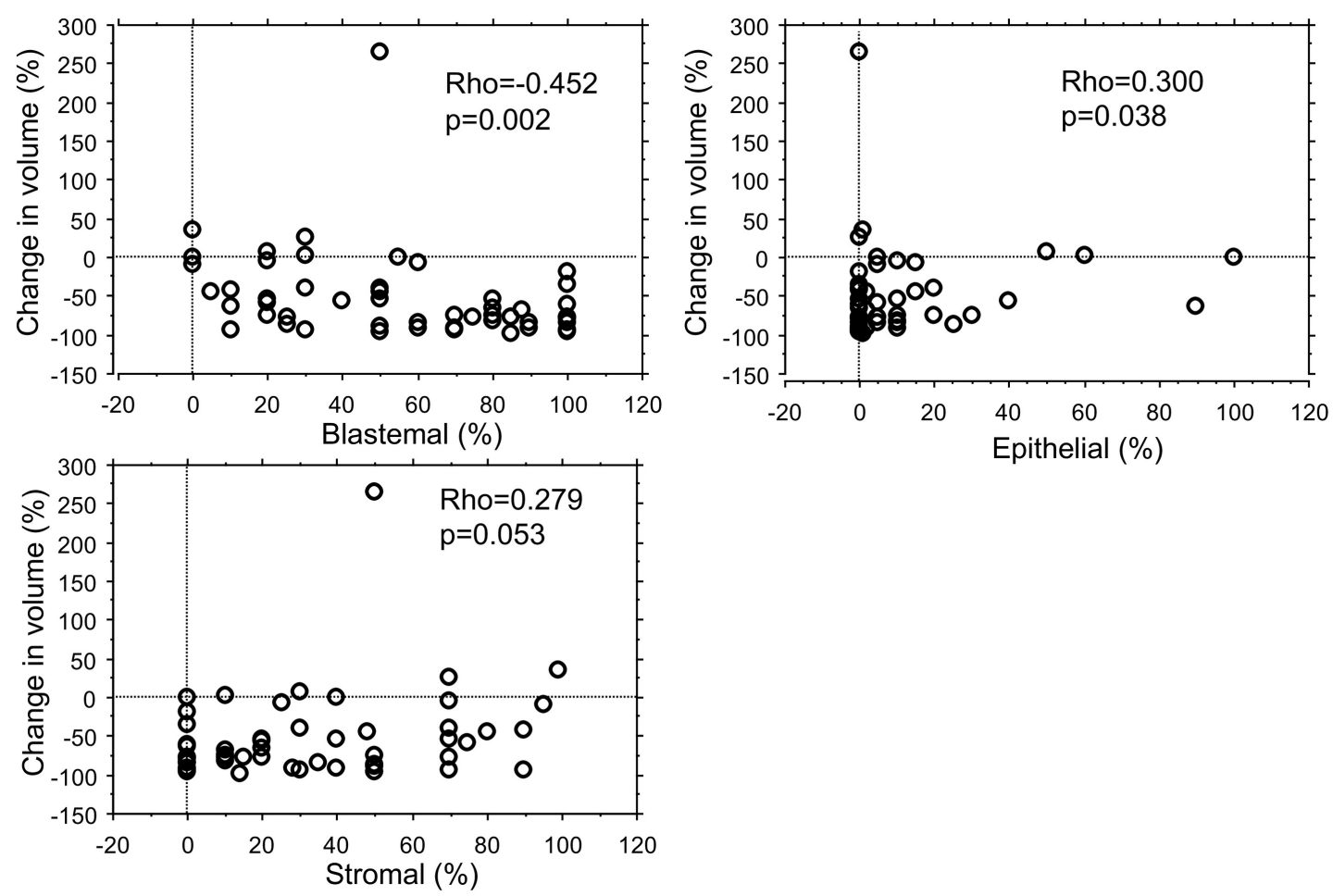

Figure 3 